\title{
Iterative Correlation-Based Controller Tuning: Application to a Magnetic Suspension System
}

\author{
A. Karimi ${ }^{1}$ L. Mišković D. Bonvin \\ Laboratoire d'Automatique, Ecole Polytechnique Fédérale de Lausanne \\ CH-1015 Lausanne, Switzerland.
}

\begin{abstract}
Iterative tuning of the parameters of a restricted-order controller using the data acquired in closed-loop operation seems to be a promising idea, especially for tuning PID controllers in industrial applications. In this paper, a new tuning approach based on decorrelation is proposed. The basic idea is to make the output error between the designed and achieved closed-loop systems uncorrelated with the reference signal. The controller parameters are calculated as the solution to correlation equations involving instrumental variables. Different choices of instrumental variables are proposed and compared via simulation. The stochastic properties of the correlation approach are compared with those of standard IFT using Monte-Carlo simulation. The proposed approach is also implemented on an experimental magnetic suspension system, and excellent performance using only a few real-time experiments is achieved.
\end{abstract}

Key words: Controller tuning, iterative methods, instrumental variables, magnetic suspension

\section{Introduction}

In model-based control design, a reduced-order model of the plant is often used to compute a controller that satisfies closed-loop specifications. But, when this controller is implemented on the real system, performance degradation will occur because of differences between the reduced-order model and the real plant. It has been shown that better performance can be obtained if a model identified in closed loop is used for controller design [?,?]. The performance may be further improved if the

$\overline{1}$ Corresponding author. Tel.: 0041(21) 6933841 Fax: 0041(21) 693 2574, e-mail: alireza.karimi@epfl.ch 
output error between the designed closed-loop system and the achieved one is minimized in the identification step. This leads to an iterative identification-for-control scheme as that proposed in [?]. In each iteration, a plant model is identified in closed-loop operation with the controller being designed on the basis of the model identified in the previous iteration. Tutorial surveys on this iterative approach can be found in $[?, ?]$.

Although the convergence of the iterative approach has not been proven, performance improvement has been observed in many applications, especially in the first few iterations. In order to cope with the convergence problem, a direct method for tuning the parameters of a restricted-order controller from closed-loop data was proposed in [?] and applied successfully to industrial plants [?,?]. This method, known as Iterative Feedback Tuning (IFT), attempts to minimize a quadratic control criterion iteratively using an unbiased estimate of the gradient without any model of the plant. In the presence of noise and a penalty on the plant input, there is typically a trade-off between closed-loop model fitting, noise attenuation and control signal limitation. A practical problem is the need for a large number of real-time experiments, which makes the IFT approach difficult (and sometimes expensive) to implement, particularly for slow industrial plants.

In this paper, a new approach for iterative controller tuning is proposed. The main idea is to modify the control objective as follows: instead of minimizing a quadratic criterion, say the 2-norm of the tracking error, one tries to make the output error between the designed and achieved closed-loop systems uncorrelated with the reference signal. This way, the achieved closed-loop system will capture the dynamics of the designed one (the controller compensates for the unmodeled plant dynamics) such that there remains no information about the reference signal in the closed-loop output error. Thus, this error will mainly contain the contribution of noise, that is uncorrelated with the reference signal. The proposed correlation approach, which is based on the instrumental variables method requires fewer real-time experiments than standard IFT . The controller chosen in this study is a two-degree of freedom RST controller.

In contrast to IFT and MRAC, the effect of noise on the closed-loop output is not minimized in this approach. It should be noted that the designed closed-loop system is typically more complex than a simple reference model for tracking since it represents a closed-loop system that meets all the specifications for tracking, control signal limitation, disturbance and noise attenuation for the initial model. Therefore, in this context, there is no interest in attenuating the output noise more than planned in the designed closed-loop system with the risk of missing the model matching performance. Hence, the objective is to make the achieved closed-loop system as close as possible to the designed one, independent of the noise characteristics. As a result the robustness properties of the designed closed-loop system will be preserved, but the performance with respect to noise attenuation will normally not change. 
The paper is organized as follows. A regression representation of the RST controller is given in the next section. Section 3 presents the correlation approach and the numerical methods used to solve the correlation equations. The choice of instrumental variables is discussed in Section 4. A relation to standard IFT is given in Section 5. Section 6 presents simulation results, while an application to an experimental magnetic suspension system is detailed in Section 7. Finally, Section ?? gives some concluding remarks.

\section{Controller Representation in Regression Form}

Let the output $y(t)$ of the true system be described by the following discrete-time model:

$$
y(t)=G\left(q^{-1}\right) u(t)+v(t)
$$

where $u(t)$ is the plant input and $v(t)$ represents a zero-mean noise. The linear time-invariant transfer operator $G\left(q^{-1}\right)$ is defined as:

$$
G\left(q^{-1}\right)=\frac{B\left(q^{-1}\right)}{A\left(q^{-1}\right)}
$$

where $B\left(q^{-1}\right)$ and $A\left(q^{-1}\right)$ are polynomials in the backward-shift operator $q^{-1}$. This system is controlled by a two-degree of freedom RST controller with the following control law:

$$
R\left(q^{-1}\right) u(t)=-S\left(q^{-1}\right) y(t)+T\left(q^{-1}\right) r(t)
$$

where

$$
\begin{aligned}
R\left(q^{-1}\right) & =1+r_{1} q^{-1}+\cdots+r_{n_{R}} q^{-n_{R}} \\
S\left(q^{-1}\right) & =s_{0}+s_{1} q^{-1}+\cdots+s_{n_{S}} q^{-n_{S}} \\
T\left(q^{-1}\right) & =t_{0}+t_{1} q^{-1}+\cdots+t_{n_{T}} q^{-n_{T}}
\end{aligned}
$$

The controller output can be presented in regression form:

$$
u(t)=\phi^{T}(\rho, t) \rho
$$

where the regressor vector $\phi(\rho, t)$ and the vector of controller parameters $\rho$, both of dimension $n_{\rho}$, are defined as follows: 


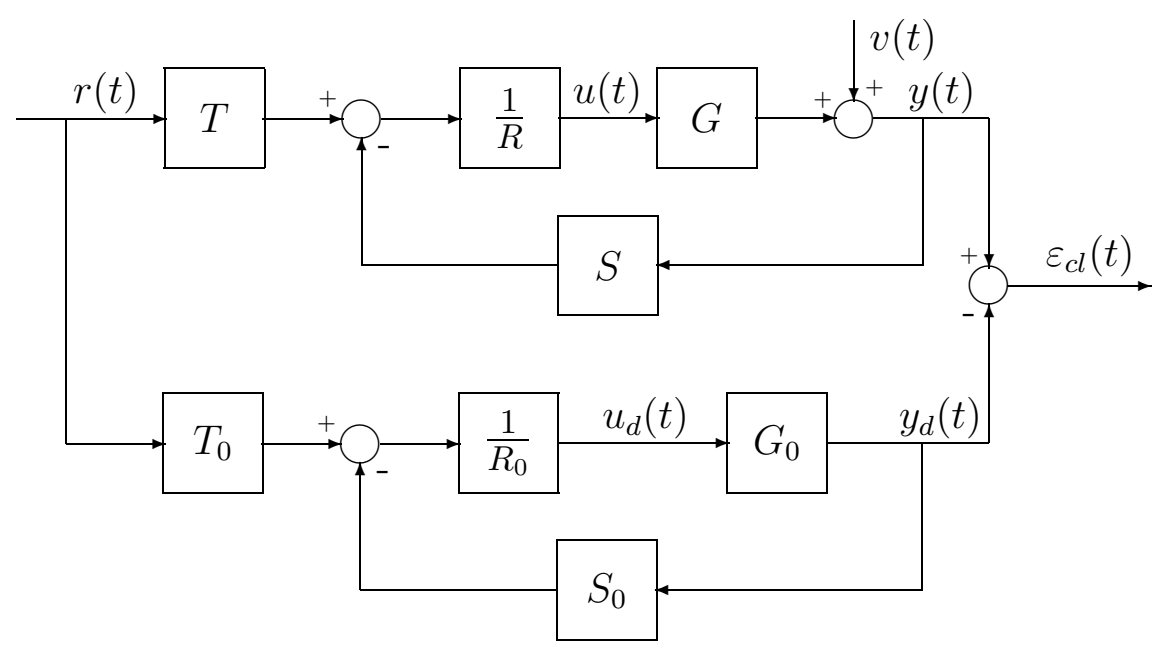

Fig. 1. Block diagram of the achieved and designed closed-loop systems

$$
\begin{aligned}
\phi^{T}(\rho, t)= & {\left[-u(t-1), \cdots-u\left(t-n_{R}\right),-y(t), \cdots-y\left(t-n_{S}\right),\right.} \\
& \left.r(t), \cdots r\left(t-n_{T}\right)\right] \\
\rho^{T}= & {\left[r_{1} \cdots r_{n_{R}}, s_{0} \cdots s_{n_{S}}, t_{0} \cdots t_{n_{T}}\right] }
\end{aligned}
$$

Since $y(t)$ depends on the controller parameters $\rho$, so does the regressor vector $\phi(\rho, t)$, with this dependency being expressed explicitly in Eq. (7).

\section{The Correlation Approach}

Figure 1 shows the block diagram of the closed-loop system with an RST controller. This diagram is often used in the context of iterative identification and control or for closed-loop output error identification [?,?]. The upper part presents the achieved closed-loop system and the lower part shows the designed closed-loop system containing the initial model of the plant $\left(G_{0}\right)$ and the initial controller $\left(R_{0}, S_{0}, T_{0}\right)$. It is assumed that the initial controller is able to meet the specifications for the designed closed-loop system, which can also be interpreted as the reference model.

The closed-loop output error is defined as:

$$
\varepsilon_{c l}(\rho, t)=y(\rho, t)-y_{d}(t)
$$

where $y(\rho, t)$ is the output of the achieved closed-loop system and $y_{d}(t)$ is the output of the designed closed-loop system or the desired output.

In iterative identification for control, the objective is to minimize the closed-loop output error $\varepsilon_{c l}(\rho, t)$ via successive closed-loop identification and model-based controller design. In the IFT approach, this error is minimized directly by tuning the parameters of the controller using closed-loop data. Inspired by the field of system 
identification [?], this work presents another way of approximating the designed closed-loop system based on the correlation approach.

Let the initial controller be applied to the real system excited by the reference signal $r(t)$ and the plant output be measured. Then, the closed-loop output error contains the effect of both modeling errors and noise. Evidently, the effect of modeling errors is correlated with the reference signal, while that of noise is not. Since the lack of control performance results essentially from the modeling error, an improved controller should be able to compensate the effect of the modeling error to the point that the closed-loop output error contains only filtered noise. Thus, a reasonable way to tune the controller parameters is to make the closed-loop output error independent of the reference signal. So, the parameters of the controller should be solution to the following $n_{\rho}$ correlation equations:

$$
f(\rho)=\frac{1}{N} \sum_{t=1}^{N} \zeta(\rho, t) \varepsilon_{c l}(\rho, t)=0
$$

where $N$ is the number of data and $\zeta(\rho, t)$ is a $n_{\rho}$-dimensional vector of instrumental variables. The instrumental variables should be correlated with the reference signal and independent of the noise.

\subsection{Iterative solution to the correlation equations}

Equation (11) is in general nonlinear and cannot be solved analytically. Iterative numerical solution is possible using the relationship:

$$
\rho_{i+1}=\rho_{i}-\gamma_{i}\left[Q\left(\rho_{i}\right)\right]^{-1} f\left(\rho_{i}\right)
$$

where $\gamma_{i}$ is the step size and $Q\left(\rho_{i}\right)$ is a square matrix of dimension $n_{\rho}$. This algorithm typically converges to a solution of Eq. (11) provided that the step size $\gamma_{i}$ is suitably chosen and $Q\left(\rho_{i}\right)$ is nonsingular [?]. Numerical problems may occur if $Q\left(\rho_{i}\right)$ is singular or close to singular (when the data are not sufficiently informative or the controller is over parameterized). The problems regarding the choice of $\gamma_{i}$ and the singularity of $Q\left(\rho_{i}\right)$ are well known in the literature and will not be discussed here [?].

If $Q\left(\rho_{i}\right)$ is chosen to be the identity matrix, there will be no singularity problem, and a simple algorithm (the substitution method) is obtained:

$$
\rho_{i+1}=\rho_{i}-\gamma_{i}\left\{\frac{1}{N} \sum_{t=1}^{N} \zeta\left(\rho_{i}, t\right) \varepsilon_{c l}\left(\rho_{i}, t\right)\right\}
$$


The iterative solution of this equation requires only one experiment per iteration (for computing $\varepsilon_{c l}\left(\rho_{i}, t\right)$ ). Since the substitution method is not very efficient near the solution, faster convergence can be obtained with the Newton-Raphson method. In this method, $Q\left(\rho_{i}\right)$ is defined as follows:

$$
Q\left(\rho_{i}\right)=\left.\frac{\partial f}{\partial \rho}\right|_{\rho=\rho_{i}}=\frac{1}{N} \sum_{t=1}^{N}\left\{\left.\frac{\partial \zeta(\rho, t)}{\partial \rho}\right|_{\rho=\rho_{i}} \varepsilon_{c l}\left(\rho_{i}, t\right)+\left.\zeta\left(\rho_{i}, t\right) \frac{\partial \varepsilon_{c l}(\rho, t)}{\partial \rho}\right|_{\rho=\rho_{i}}\right\}
$$

An accurate value of this Jacobian matrix cannot be computed because the derivative of $\varepsilon_{c l}(\rho, t)$ with respect to $\rho$ is unknown. However, precise knowledge of the Jacobian matrix is not important in practice as a good estimate of this matrix is only required in the neighborhood of the solution [?].

\subsection{Jacobian estimation}

The first term in Eq. (14) is close to zero because the derivatives of the instrumental variables are uncorrelated with the closed-loop output error near the solution. Neglecting this term gives:

$$
Q\left(\rho_{i}\right)=\left.\frac{\partial f}{\partial \rho}\right|_{\rho=\rho_{i}} \approx \frac{1}{N} \sum_{t=1}^{N} \zeta\left(\rho_{i}, t\right) \psi^{T}\left(\rho_{i}, t\right)
$$

where $\psi(\rho, t)$ is the gradient of the closed-loop output (error) with respect to the parameter vector:

$$
\psi^{T}(\rho, t)=\frac{\partial \varepsilon_{c l}(\rho, t)}{\partial \rho}=\frac{\partial y(\rho, t)}{\partial \rho}
$$

The input and output of the plant in the closed-loop system are described as:

$$
\begin{aligned}
& y(\rho, t)=\frac{B\left(q^{-1}\right) T\left(q^{-1}\right)}{P\left(q^{-1}\right)} r(t)+\frac{A\left(q^{-1}\right) R\left(q^{-1}\right)}{P\left(q^{-1}\right)} v(t) \\
& u(\rho, t)=\frac{A\left(q^{-1}\right) T\left(q^{-1}\right)}{P\left(q^{-1}\right)} r(t)-\frac{A\left(q^{-1}\right) S\left(q^{-1}\right)}{P\left(q^{-1}\right)} v(t)
\end{aligned}
$$

where $P\left(q^{-1}\right)$ is the closed-loop characteristic polynomial:

$$
P\left(q^{-1}\right)=A\left(q^{-1}\right) R\left(q^{-1}\right)+B\left(q^{-1}\right) S\left(q^{-1}\right)
$$

For ease of notation, $q^{-1}$ is omitted in the sequel of the paper. The derivatives of $y$ with respect to the parameters of $R$ are computed as follows [?]: 


$$
\begin{aligned}
\frac{\partial y}{\partial r_{j}} & =\frac{-q^{-j} A B T}{P^{2}} r(t)+\frac{q^{-j} A P-q^{-j} A A R}{P^{2}} v(t) \\
& =\frac{-B q^{-j}}{P}\left[\frac{A T}{P} r(t)-\frac{A S}{P} v(t)\right] \\
& =\frac{-B}{P} u(t-j) \quad j=1, \ldots, n_{R}
\end{aligned}
$$

In the same way, the derivatives of $y$ with respect to the other controller parameters can be computed:

$$
\begin{array}{lc}
\frac{\partial y}{\partial s_{j}}=\frac{-B}{P} y(t-j) & j=0, \ldots, n_{S} \\
\frac{\partial y}{\partial t_{j}}=\frac{B}{P} r(t-j) & j=0, \ldots, n_{T}
\end{array}
$$

Thus, the gradient of $y$ with respect to $\rho$ can be represented in terms of the regressor vector $\phi$ filtered by $\frac{B}{P}$ :

$$
\psi^{T}(\rho, t)=\frac{\partial y(\rho, t)}{\partial \rho}=\frac{B}{P} \phi^{T}(\rho, t)
$$

Although the gradient vector can be obtained by two additional experiments on the real system, as this is done in IFT, it can also be computed by filtering the regressor vector using the identified model of the closed-loop system $\left(\frac{\hat{B}}{\hat{P}}\right)$. Thus, the following estimate of the Jacobian matrix can be used:

$$
Q\left(\rho_{i}\right)=\frac{1}{N} \sum_{t=1}^{N} \zeta\left(\rho_{i}, t\right) \bar{\psi}^{T}\left(\rho_{i}, t\right)
$$

where

$$
\bar{\psi}^{T}(\rho, t)=\frac{\hat{B}}{\hat{P}} \phi^{T}(\rho, t)
$$

It should be mentioned that the required closed-loop filter $\frac{\hat{B}}{\hat{P}}$ can be identified using open-loop identification methods. This technique has been proposed in [?] for the standard IFT method to overcome practical implementation problems due to large errors in the initial iterations. But it could not be continued up to the final iterations because, with an approximate gradient, the convergence of the controller parameters to the optimal values cannot be proven. However, in the proposed approach, the gradient appears only in the Jacobian matrix, i.e., it affects only the convergence speed and not the convergence itself. In other words, even with an approximate Jacobian, the controller parameters converge to the solution of the correlation equations (if the 
step size is properly chosen). Furthermore, the estimation of the Jacobian matrix in Eq. (26) is asymptotically not affected by noise (because $\zeta(\rho, t)$ is uncorrelated with $\bar{\psi}(\rho, t))$ and if a high-order FIR model of the closed-loop system is used for filtering the regressor vector (see Eq. (27)), a reliable unbiased estimate of the Jacobian matrix will be obtained.

\subsection{Fixed terms in the controller}

In order to preserve certain properties of the initial controller (e.g. integral action), fixed terms can be considered in the $\mathrm{R}$ and $\mathrm{S}$ polynomials (i.e., $R=R^{\prime} R_{\text {fix }}$ and $\left.S=S^{\prime} S_{f i x}\right)$. In this case, the input $u(t)$ and the output $y(t)$ of the system are replaced in $\psi(\rho, t)$ by $u^{\prime}(t)=R_{f i x} u(t)$ and $y^{\prime}(t)=S_{f i x} y(t)$, respectively. Then, $R^{\prime}$ and $S^{\prime}$ are computed using the iterative algorithm and later multiplied by the fixed terms to obtain the controller polynomials $R$ and $S$.

\section{Choice of Instrumental Variables}

It is well known that the instrumental variables should be correlated with the regressor vector and uncorrelated with noise. The choice of instrumental variables can affect the convergence speed and the numerical problems encountered with the Newton-Raphson method [?].

A trivial choice of instrumental variables is a vector containing $n_{\rho}$ delayed values of the reference signal:

$$
\zeta^{T}(t)=\left[r(t-1), r(t-2), \ldots, r\left(t-n_{\rho}\right)\right]
$$

This choice needs no computational effort and, since the instruments are not functions of $\rho$, the first term in Eq. (14) is always zero. However, this choice leads to an iterative algorithm that may converge very slowly.

An idealized choice of instruments is a noise-free estimate of the gradient $\psi(\rho, t)$ that is based only on the reference signal [?]. This makes $Q(\rho)$ as close as possible to a positive semi-definite matrix. Then, standard regularization techniques can be employed to make this matrix always positive definite. The gradient $\psi(\rho, t)$ can be estimated by filtering (using the closed-loop filter $\frac{\hat{B}}{\hat{P}}$ ) a noise-free estimate of the regressor vector that can be obtained in two different ways. The first approach is based on identified models, and the corresponding Iterative Correlation-based Tuning will be labeled ICT-IM. The second approach uses the designed-output, leading to the acronym ICT-DO. The choices are explicated below: 
- Identified models of the closed-loop system (ICT-IM):

$$
\zeta_{I M}(\rho, t)=\hat{\psi}(\rho, t)=\frac{\hat{B}}{\hat{P}} \hat{\phi}(\rho, t)
$$

where

$$
\begin{aligned}
\hat{\phi}^{T}(\rho, t)= & {\left[-\hat{u}(t-1) \cdots-\hat{u}\left(t-n_{R}\right),-\hat{y}(t) \cdots-\hat{y}\left(t-n_{S}\right),\right.} \\
& \left.r(t) \cdots r\left(t-n_{T}\right)\right]
\end{aligned}
$$

and

$$
\begin{aligned}
& \hat{u}(t)=\frac{\hat{A} T}{\hat{P}} r(t) \\
& \hat{y}(t)=\frac{\hat{B} T}{\hat{P}} r(t)
\end{aligned}
$$

The closed-loop models $\frac{\hat{A} T}{P}$ and $\frac{\hat{B} T}{P}$ can be identified using open-loop identification methods or they may be computed using the plant model identified in closed loop [?] and the known controller.

- Designed output (ICT-DO):

$$
\zeta_{D O}(\rho, t)=\psi_{d}(\rho, t)=\frac{\hat{B}}{\hat{P}} \phi_{d}(\rho, t)
$$

where

$$
\begin{aligned}
\phi_{d}^{T}(\rho, t)= & {\left[-u_{d}(t-1) \cdots-u_{d}\left(t-n_{R}\right),-y_{d}(t) \cdots-y_{d}\left(t-n_{S}\right),\right.} \\
& \left.r(t) \cdots r\left(t-n_{T}\right)\right]
\end{aligned}
$$

and

$$
u_{d}(t)=\frac{T}{R} r(t)-\frac{S}{R} y_{d}(t)
$$

Notice that the vector $\phi_{d}(\rho, t)$ is independent of noise and its computation does not require the knowledge of the plant model.

This two choices of instrumental variables will be compared via Monte-Carlo simulation in Section 6. 


\section{$5 \quad$ Relation to Standard IFT}

To facilitate comparison, the standard IFT method will be presented in regression form. The control criterion is defined as:

$$
J(\rho)=\frac{1}{2} \sum_{t=1}^{N} \varepsilon_{c l}^{2}(\rho, t)=\frac{1}{2} \sum_{t=1}^{N}\left[y(\rho, t)-y_{d}(t)\right]^{2}
$$

with the gradient equation:

$$
\frac{\partial J}{\partial \rho}=\sum_{t=1}^{N} \frac{\partial y}{\partial \rho} \varepsilon_{c l}(\rho, t)=\sum_{t=1}^{N} \psi^{T}(\rho, t) \varepsilon_{c l}(\rho, t)=0
$$

This equation is analogues to the correlation equation (11) in which all terms are perfectly known from one experiment. But, since $\psi(\rho, t)$ in Eq. (37) is not available, two experiments on the real system are performed in order to compute the model-free unbiased estimate $\tilde{\psi}(\rho, t)$ and another experiment for computing $\varepsilon_{c l}(\rho, t)$.

Now, consider the Hessian matrix:

$$
H(\rho)=\frac{\partial^{2} J}{\partial \rho^{2}}=\sum_{t=1}^{N}\left\{\frac{\partial \psi}{\partial \rho} \varepsilon_{c l}(\rho, t)+\psi(\rho, t) \psi^{T}(\rho, t)\right\}
$$

Since this matrix is not available, the following approximation can be used:

$$
\tilde{H}(\rho)=\sum_{t=1}^{N} \tilde{\psi}(\rho, t) \tilde{\psi}^{T}(\rho, t)
$$

This approximation is biased [?] and generally decreases the convergence rate in the neighborhood of the solution.

The iterative equation for standard IFT in regression form is:

$$
\rho_{i+1}=\rho_{i}-\gamma_{i}\left\{\sum_{t=1}^{N} \tilde{\psi}\left(\rho_{i}, t\right) \tilde{\psi}^{T}\left(\rho_{i}, t\right)\right\}^{-1} \sum_{t=1}^{N} \tilde{\psi}\left(\rho_{i}, t\right) \varepsilon_{c l}\left(\rho_{i}, t\right)
$$

It is interesting to compare this equation with that of the correlation approach with the idealized instrumental variables $\zeta_{I M}(\rho, t)=\hat{\psi}(\rho, t)$ :

$$
\rho_{i+1}=\rho_{i}-\gamma_{i}\left\{\sum_{t=1}^{N} \hat{\psi}\left(\rho_{i}, t\right) \bar{\psi}^{T}\left(\rho_{i}, t\right)\right\}^{-1} \sum_{t=1}^{N} \hat{\psi}\left(\rho_{i}, t\right) \varepsilon_{c l}\left(\rho_{i}, t\right)
$$


It is clear that these two equations are very similar. In fact, they give asymptotically the same results in the absence of noise. However, in the presence of noise the methods are clearly different. Yet, they will asymptotically converge under the same conditions to the solution of their respective equations (gradient equation for IFT and correlation equation for the proposed approach). Regarding the estimation of the Hessian and Jacobian matrices, the former is a biased estimate, while the later is unbiased (since $\hat{\psi}$ is uncorrelated with the noisy part of $\bar{\psi}$ ). Thus, even for the same number of iterations (three times more experiments for the IFT), it is expected that the correlation method give faster convergence. This will be illustrated by a Monte-Carlo simulation in the next section.

\section{Simulation results}

Extensive Monte-Carlo simulations have been performed in order to investigate the basic features of the two algorithms (standard IFT and correlation approach). Two different simulation studies are presented below: Simulation 1 investigates the choice of instrumental variables, while Simulation 2 compares the proposed correlation approach to IFT.

The simulated test system is given by the following transfer function:

$$
G=\frac{q^{-1}+0.5 q^{-2}}{\left(1-1.5 q^{-1}+0.7 q^{-2}\right)\left(1-0.5 q^{-1}\right)}
$$

The dominant dynamics is characterized by one very oscillatory mode. The initial controller is calculated by pole placement using the following reduced-order model:

$$
G_{0}=\frac{8 q^{-1}+2.6 q^{-2}}{1-1.2 q^{-1}+0.6 q^{-2}}
$$

This model was purposely chosen as a poor approximation to the true plant $G$. The first design specification is to obtain a closed-loop system that preserves the natural frequency of the dominant mode of the open-loop system, but with a damping factor of 0.95 . As a second requirement, the polynomial $R_{0}$ should contain the fixed factor $R_{f i x}\left(q^{-1}\right)=1-q^{-1}$ in order to provide integral action. The precompensator $T_{0}\left(q^{-1}\right)$ is chosen to obtain unity closed-loop gain. The initial controller reads:

$$
\begin{aligned}
R_{0}\left(q^{-1}\right) & =1-0.7238 q^{-1}-0.2762 q^{-2} \\
S_{0}\left(q^{-1}\right) & =0.1189-0.1565 q^{-1}+0.0637 q^{-2} \\
T_{0}\left(q^{-1}\right) & =0.0261
\end{aligned}
$$




\subsection{Simulation 1: Choice of instrumental variables}

Two different choices of instrumental variables for the correlation approach are compared. The first choice corresponds to an estimate of the gradient that is based on identified closed-loop models (ICT-IM, Eq. (29)). The closed-loop models used for filtering $\left(\frac{\hat{A} T}{\hat{P}}, \frac{\hat{B} T}{\hat{P}}, \frac{\hat{B}}{\hat{P}}\right)$ are computed using a plant model $\left(\frac{\hat{B}}{\hat{A}}\right)$ and the known controller. The plant model is identified in closed loop using a second-order ARMAX structure. Model-order mismatch is introduced to show that an approximate model can be used in the calculations without significant loss in performance. The second choice is an estimate of the gradient based on the designed output (ICT-DO, Eq. (33)). The closed-loop filter $\left(\frac{\hat{B}}{\hat{P}}\right)$ is computed in the same manner as for the first choice. In all iterations, the constant step size $\gamma_{i}=0.5$ is used.

To compare these two choices, 300 simulations are performed. For each simulation run, the optimization is carried out over 9 iterations. Each experiment is performed with a different realization of the measurement noise, which is generated as a zeromean, stationary, white Gaussian sequence with a standard deviation $\sigma= \pm 0.025$. The reference signal is a unit step.

The results of this simulation study are presented in Fig. 2. Let the averaged correlation index, $A C I$, be defined as:

$$
A C I(i)=\frac{1}{n_{s}} \sum_{k=1}^{n_{s}}\left\|f_{k}\left(\rho_{i}\right)\right\|_{2}
$$

where $n_{s}$ denotes the number of simulations, $k$ is the simulation index and $i$ denotes the iteration number. The evolution of the ACIs obtained with ICT-IM (solid line) and ICT-DO (dashed line) are given in Fig. 2. The curves indicate that both variants of instrumental variables succeed to reduce the averaged correlation index $A C I$.

\subsection{Simulation 2: ICT-IM versus IFT}

In the second simulation study, the averaged criterion, $A C J$, is introduced in order to compare the standard IFT algorithm and ICT-IM:

$$
A C J(i)=\frac{1}{n_{s}} \sum_{k=1}^{n_{s}} J^{(k)}\left(\rho_{i}\right)
$$

where $J^{(k)}$ is the IFT control criterion defined in Eq. (36) for the k-th Monte-Carlo simulation. Although the criterion (36) is not minimized by the ICT-IM method, it is reduced because this method tries to suppress the correlated part of the closed-loop 


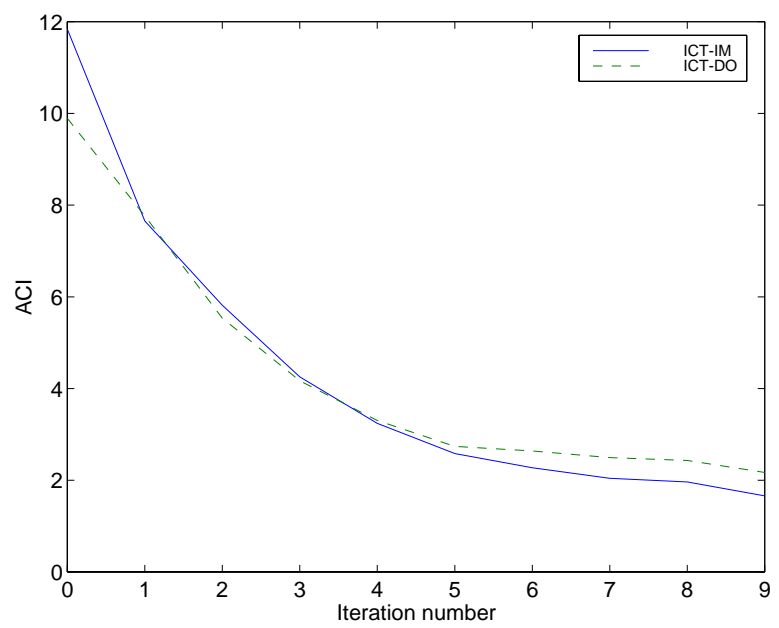

Fig. 2. Correlation indices averaged over 300 noise realizations for ICT-IM (solid) and ICT-DO (dashed) over 9 iterations

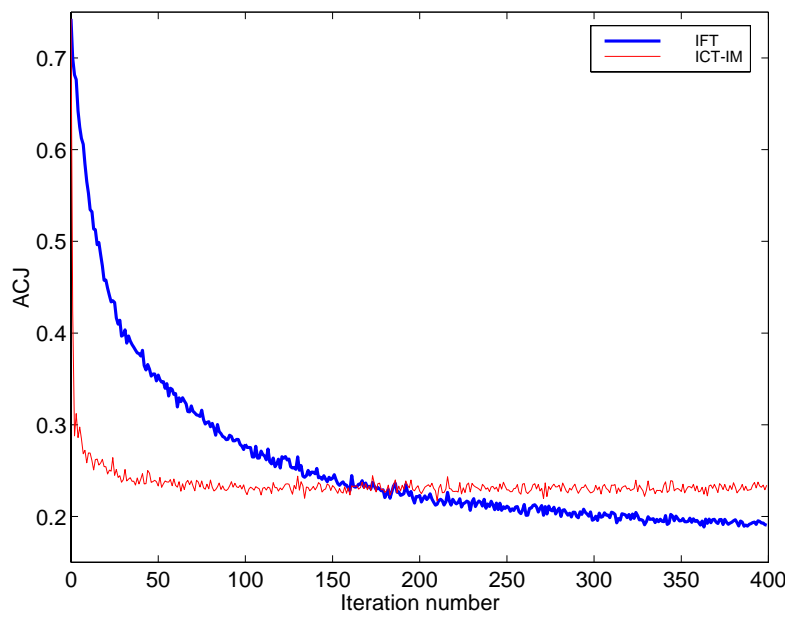

Fig. 3. Criterion J averaged over 100 noise realizations for IFT (thick line) and ICT-IM (thin line) over 400 iterations

output error (the closed-loop output error contains the noise and the contribution of the modelling errors that are correlated with the reference signal).

In the first part, in order to compare the asymptotic behavior of two algorithms, 100 Monte-Carlo simulations are performed, each with 400 iterations. The simulation conditions (measurement noise, reference signal and designed output) are the same as those in Simulation 1. A Gauss-Newton update direction with step size $\gamma_{i}=1$ is used in the IFT procedure. Penalty on the control effort is not incorporated in the criterion. The step size $\gamma_{i}=1$ is also chosen for ICT-IM. The two approaches are compared in terms of $A C J$ in Fig. 3. It is clear from the figure that IFT, as expected, converges to a lower value of the criterion $A C J$ than ICT-IM. However, in the first iterations ICT-IM reduces the criterion much faster. 


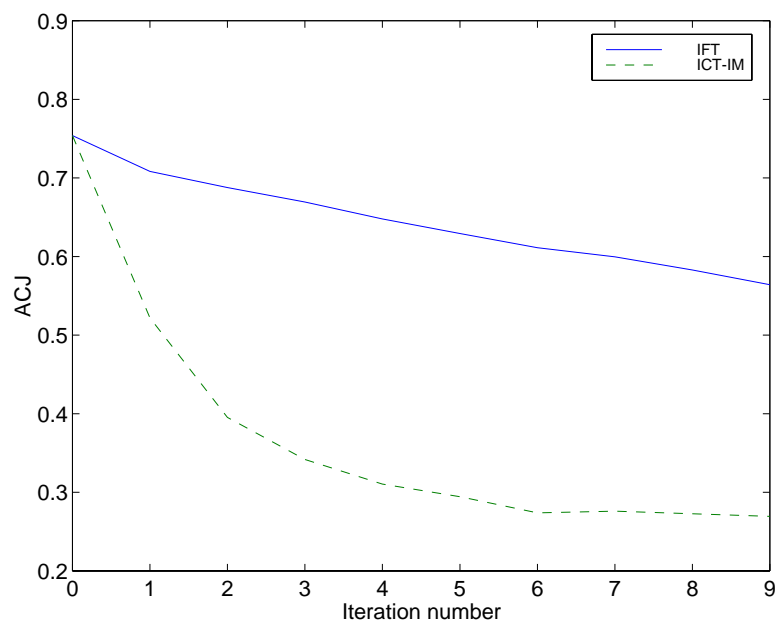

Fig. 4. Criterion J averaged over 500 noise realizations for IFT (solid) and ICT-IM (dashed) over 9 iterations

It is of interest in practice to use only a limited number of iterations. Thus, in the second part, 500 simulations are performed, each with 9 iterations, i.e., 27 experiments in the case of IFT and 9 experiments for ICT-IM. The simulation conditions (measurement noise, reference signal and designed output) are the same as those in the first part. Fig. 4 shows that ICT-IM reaches the final value after 6 iterations, while the cost with IFT decreases at a much slower rate. Notice the remarkable speed of convergence and the low $A C J$ value for ICT-IM.

\section{Application to a Magnetic Suspension System}

In this section, the effectiveness of the correlation approach is demonstrated experimentally on a nonlinear and unstable magnetic suspension system. First, the experimental set up is described. Then, the design of the initial controller is explained, and the results of iterative tuning using the proposed method and standard IFT are shown.

\subsection{The magnetic suspension system}

The magnetic suspension system is illustrated in Fig. 5. A ferromagnetic sphere is suspended in the air using a magnetic force to compensate for the gravitational force. The actuator in the system is a solenoid which produces a magnetic force when current flows through the coil. The manipulated input $u$ is voltage to a U/I (voltage-to-current) converter that supplies current to the coil. The position of the sphere is measured by an optical sensor: variations in the position of the sphere change the intensity of the measured light, which in turn changes the voltage in the 


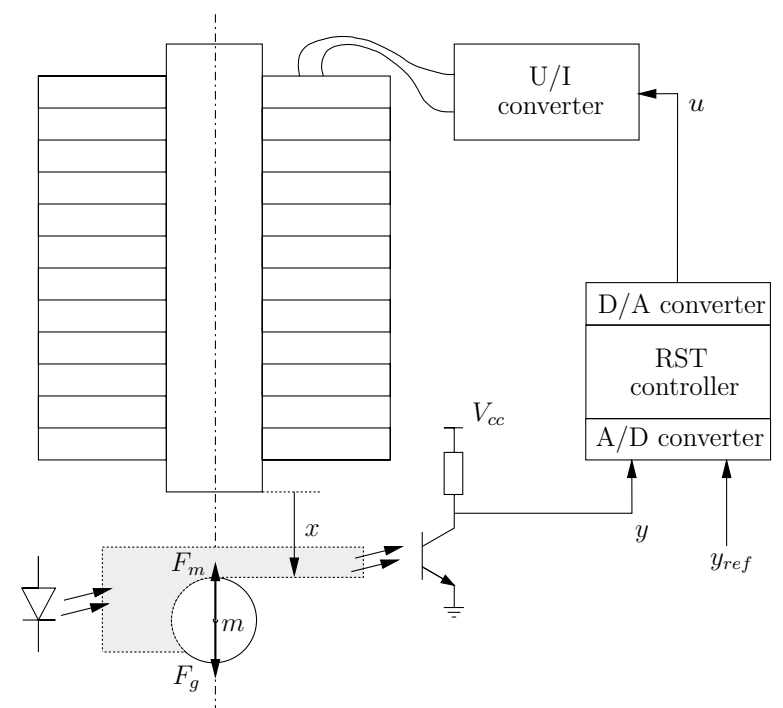

Fig. 5. Magnetic suspension system

measurement circuit. The output $y$ is the measured voltage corresponding to the position of the sphere. The system is controlled by a Sun station via an I/O board. A model of the system is needed to design the initial controller. A momentum balance gives:

$$
m \ddot{x}=F_{g}-F_{m}
$$

where $m$ and $x$ denote the mass of the sphere and its position, respectively, $F_{g}=m g$ denotes the gravitational force, while $F_{m}$ denotes the magnetic force. The latter can be expressed as $F_{m}=-\frac{1}{2} \frac{d L}{d x} i^{2}$, where $L$ is the inductance that decreases with $x$. The dynamics of the current $i$ can be approximated well by a first-order system. The current loop is controlled by an analog high-gain controller and, therefore, its dynamics can be neglected. Since $\frac{d L}{d x}$ depends nonlinearly on $x$, it is necessary to linearize the system dynamics around a stationary point. The resulting transfer function is given by:

$$
G(s)=\frac{K_{u i}}{\tau_{u i} s+1} \frac{b}{s^{2}-a}
$$

where $K_{u i}$ and $\tau_{u i}$ denote the gain and the time constant of the U/I converter, respectively. Note that the parameters $a$ and $b$ vary with the linearization point. The linearization of Eq. (50) around the stationary point $x=0.0525 \mathrm{~m}$ gives the values of parameters that are shown in Table 1.

The magnetic suspension system is unstable and requires a controller for stabilization. The reference input, $y_{r e f}$, is the voltage corresponding to the desired sphere position. The system is sampled at the frequency $f_{s}=100 \mathrm{~Hz}$. 
Table 1

\begin{tabular}{|c|c|c|c|}
\hline \hline$K_{u i}$ & $\tau_{u i}$ & $b$ & $a$ \\
\hline 0.1033 & 0.0173 & 15749.4 & 1238 \\
\hline \hline
\end{tabular}

Model parameters obtained after linearization around the stationary point

It should be emphasized that this model will not be used in the iterative scheme, and is computed only for determining a reasonable reference model and the initial controller.

\subsection{Experimental results}

For implementation purposes, $u$ and $y$ correspond to deviations from a stationary operating point $\left(u_{0}, y_{0}\right)$. The value of $u_{0}$ is manually adjusted to make $y_{0}$ approach $r_{0}$, the stationary value of the reference signal, without static offset. A square wave signal is chosen as the reference $r$ with an amplitude of $0.3 \mathrm{~V}$ and a period of $1 \mathrm{~s}$. The data length used in each experiment is $4 \mathrm{~s}$.

Discretization of Eq. (50) with the sampling rate $f_{s}$ gives the following discrete-time model:

$$
G_{0}\left(q^{-1}\right)=\frac{0.0137 q^{-1}+0.0481 q^{-2}+0.0103 q^{-3}}{1.0-2.6861 q^{-1}+2.1922 q^{-2}-0.5610 q^{-3}}
$$

The initial controller is in the form of a two-degree of freedom RST controller. Pole placement is used to compute the coefficients of the polynomials $R_{0}\left(q^{-1}\right)$ and $S_{0}\left(q^{-1}\right)$, while the precompensator $T_{0}\left(q^{-1}\right)$ is chosen to obtain unity closed-loop gain. The initial RST polynomials are:

$$
\begin{aligned}
R_{0}\left(q^{-1}\right) & =1+0.6859 q^{-1}+0.1631 q^{-2} \\
S_{0}\left(q^{-1}\right) & =21.8601-26.7734 q^{-1}+8.1504 q^{-2} \\
T_{0}\left(q^{-1}\right) & =1.8297
\end{aligned}
$$

Since $G_{0}$ is only a rough approximation of the real system in the vicinity of the operating point, the designed and achieved closed-loop responses differ considerably as seen in Figure ??. In order to improve the closed-loop performance, and to make the responses of the achieved and designed closed-loop systems as close as possible, two iterative tuning procedures are used and compared.

The standard IFT scheme with the following design choices is applied first: GaussNewton direction, step size $\gamma_{i}=1$, control weight $\lambda=0$. At each step of the iterative design, a second experiment is performed by feeding back, at the reference input, the error between the reference signal and the output of the first experiment. The 


\begin{tabular}{|c|ccccccccc|}
\hline \hline Iteration & 0 & 1 & 2 & 3 & 4 & 5 & 6 & 7 & 8 \\
\hline IFT & 1.9454 & 0.6722 & 0.3246 & 0.1919 & 0.1455 & 0.1671 & 0.2507 & 0.1483 & 0.1217 \\
\hline ICT-IM & 1.9454 & 0.4680 & 0.1589 & 0.1126 & 0.1303 & 0.0830 & 0.0590 & 0.1156 & 0.0920 \\
\hline
\end{tabular}

Table 2

Observed sum of squared output errors of 9 successive controllers for both IFT and ICT-IM

closed-loop response obtained after 8 iterations (24 experiments) is shown in Figure ?? (solid line), with the designed response shown in dashed line. The resulting closedloop system has dynamics and static gain very close to the desired ones. The cost $J$ is reduced by $93 \%$.

Then, starting with the same initial controller and using the input-output data of the first experiment in the first iteration of the IFT procedure, a new controller is calculated using the correlation approach. In this way, it is ensured that both iterative tuning techniques have the same initial conditions. The step size is also fixed at $\gamma_{i}=1$. An ARMAX structure $\left(n_{a}=3, n_{b}=3, n_{c}=3, n_{k}=1\right)$ is considered for the plant model and no special effort is carried out for order estimation or model validation in order to show that the algorithm is not very sensitive to modeling error. After 6 iterations, this procedure leads to the closed-loop response shown in Figure ?? (solid line). A comparison with the initial response (Fig. ??) shows that ICT-IM improves the performance significantly.

Although the correlation approach calculates the parameters of the controller by solving the correlation equations (11), it also reduces the sum of squared output error. Table ?? compares the observed sum of squared output errors of 9 successive controllers for both IFT and ICT-IM. It is evident that the correlation approach converges faster than standard IFT. This may be explained intuitively by the fact that the estimation of the Hessian is biased, whereas the estimation of the Jacobian is not.

\section{Conclusions}

A new iterative method for tuning controller parameters based on the correlation approach has been presented. It has been shown that making the output error between the achieved and designed closed-loop systems uncorrelated with the reference signal, can be used as an objective for controller tuning. The resulting controller preserves the designed robustness and performance for the achieved closed loop, independently of the noise characteristics. The proposed iterative method needs only one experiment per iteration and converges within a few iterations. The algorithm requires an approximate model of the plant for computing the gradient of the output error. However, the method is not sensitive to modeling errors, since this gradient is not involved in the correlation equations but only in their derivatives with respect 


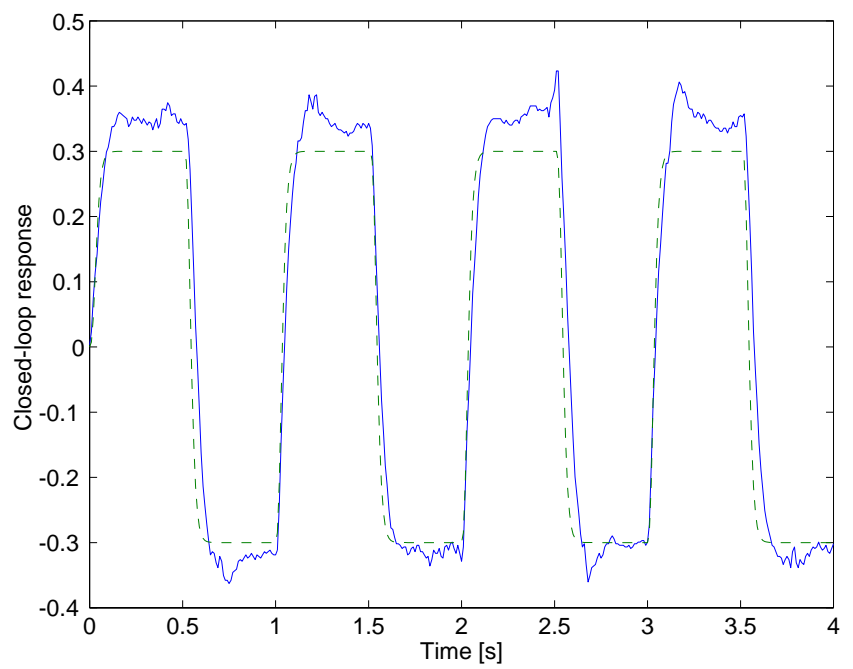

Fig. 6. Closed-loop response achieved with the initial RST controller (solid) and designed response (dashed)

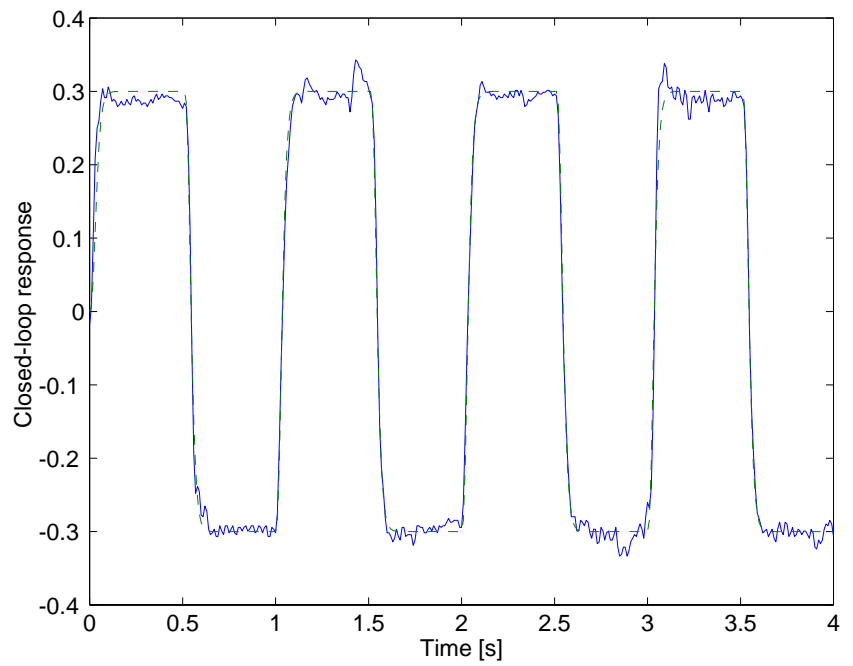

Fig. 7. Closed-loop response achieved with the IFT after 8 iterations (solid), and designed response (dashed)

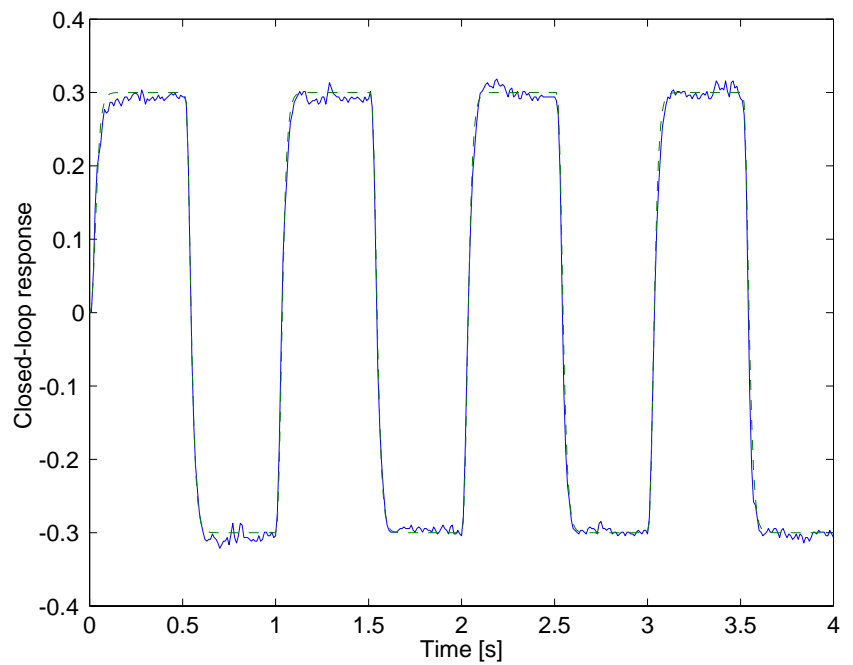

Fig. 8. Closed-loop response achieved with the ICT-IM after 6 iterations (solid), and designed response (dashed) 
to the parameters $\rho$. Thus, inaccuracies in the gradient affect only the convergence speed and not the convergence itself. Excellent performance has been obtained in simulation and real-time application to a magnetic suspension system.

\section{Acknowledgement}

The authors wish to express their thanks to David Hoover and Sergio Valentinotti for their support during the real-time experiments.

\section{References}

[1] K. J. Astrom and B. Wittenmark. Adaptive Control. Addison-Wesley, 1989.

[2] F. De Bruyne and P. Carrette. Synthetic generation of the gradient for an iterative controller optimization method. In 4th European Control Conference, Paper THA-F2 1997.

[3] M. Gevers. Towards a joint design of identification and control? In H. L. Trentelman and J.C. Willems, editors, Essays on Control, Perspectives in the Theory and its Applications. Birkhäuser, Boston, USA, 1993.

[4] M. Gevers and L. Ljung. Optimal experiment designs with respect to the intended model application. Automatica, 22(5):543-554, 1986.

[5] H. Hjalmarsson and M. T. Cameron. Iterative feedback tuning of controllers in cold rolling mills. In 14th Triennial World Congress IFAC, pages 445-450, Beijing, P. R. China, 1999.

[6] H. Hjalmarsson, M. Gevers, F. De Bruyne, and J. Leblond. Identification for control: Closing the loop gives more accurate controllers. In Conference on Decision and Control, pages 4150-4155, Lake Buena Vista, FL, USA, 1994.

[7] H. Hjalmarsson, M. Gevers, S. Gunnarsson, and O. Lequin. Iterative feedback tuning: Theory and application. IEEE Control Systems Magazine, pages 26-41, 1998.

[8] H. Hjalmarsson, S. Gunnarsson, and M. Gevers. A convergent iterative restricted complexity control design scheme. In 33rd IEEE-CDC, December 1994.

[9] I. D. Landau and A. Karimi. An output error recursive algorithm for unbiased identification in closed loop. Automatica, 33(5):933-938, May 1997.

[10] I. D. Landau and A. Karimi. Recursive algorithms for identification in closed loop a unified approach and evaluation. Automatica, 33(8):1499-1523, August 1997.

[11] L. Ljung. System Identification - Theory for the User. Prentice Hall, NJ, USA, 1987. 
[12] R. R. Schrama. Accurate identification for control: The necessity of an iterative scheme. IEEE Transactions on Automatic Control, 37(7):991-994, July 1992.

[13] T. Söderström and P. Stoica. Instrumental variable methods for system identification. In A. V. Balakrishnan and M. Thoma, editors, Lecture Notes in Control and Information Science. Springer-Verlag, Berlin, 1983.

[14] P. M. J. Van den Hof and R. R. Schrama. Identification and control - Closed-loop issues. Automatica, 31(12):1751-1770, December 1995. 


\section{List of Figures and Tables}

- Figure 1: Block diagram of the achieved and designed closed-loop systems

- Figure 2: Correlation indices averaged over 300 noise realizations for ICT-IM (solid) and ICT-DO (dashed) over 9 iterations

- Figure 3: Criterion J averaged over 100 noise realizations for IFT (thick line) and ICT-IM (thin line) over 400 iterations

- Figure 4: Criterion J averaged over 500 noise realizations for IFT (solid) and ICT-IM (dashed) over 9 iterations

- Figure 5: Magnetic suspension system

- Figure 6: Closed-loop response achieved with the initial RST controller (solid) and designed response (dashed)

- Figure 7: Closed-loop response achieved with the IFT after 8 iterations (solid), and designed response (dashed)

- Figure 8: Closed-loop response achieved with the ICT-IM after 6 iterations (solid), and designed response (dashed)

- Table 1: Model parameters obtained after linearization around the stationary point

- Table 2: Observed sum of squared output errors of 9 successive controllers for both IFT and ICT-IM 